\title{
Unconventional thermoelectric effect in superconductors that break time-reversal symmetry
}

\author{
Mihail Silaev, Julien Garaud, and Egor Babaev \\ Department of Theoretical Physics, KTH-Royal Institute of Technology, Stockholm, SE-10691 Sweden
}

(Dated: September 16, 2021)

\begin{abstract}
We demonstrate that superconductors which break time-reversal symmetry can exhibit thermoelectric properties, which are entirely different from the Ginzburg mechanism. As an example, we show that in the $s+i s$ superconducting state there is a reversible contribution to thermally induced supercurrent, whose direction is not invariant under time-reversal operation. Moreover in contrast to Ginzburg's mechanism it has a singular behavior near the time-reversal symmetry breaking phase transition. The effect can be used to confirm or rule out the $s+i s$ state, which is widely expected to be realized in pnictide compounds $\mathrm{Ba}_{1-x} \mathrm{~K}_{x} \mathrm{Fe}_{2} \mathrm{As}_{2}$ and stoichiometric LiFeAs.
\end{abstract}

PACS numbers: $74.25 . f g, 74.20 . \mathrm{Rp}$

Thermoelectric effects in superconductors, and their fundamental importance, were first discussed 70 years ago by Ginzburg [1,2]. Currently there is a revival of interest in this topic [3-6]. These effects originate in charge transfer by thermal quasiparticles [7], which is compensated by the counterflow of superconducting current $\boldsymbol{j}_{s}=-b_{n} \boldsymbol{\nabla} T$ (where $b_{n}$ is the normal component thermoelectric coefficient). As it is determined by the dissipative normal current, such a thermally induced supercurrent is irreversible since $\boldsymbol{j}_{s}$ changes sign under the time-reversal transformation while $b_{n}$ and $\nabla T$ remain invariant. We point out here, that multicomponent superconductors which break the time-reversal symmetry, have entirely different reversible thermoelectric response. Namely, there is a generic contribution to the thermally induced supercurrent, whose direction is not preserved under time-reversal operation. Besides, that contribution can be orders of magnitude larger than the ordinary one and, in contrast to Ginzburg mechanism, be present even at low temperatures. More precisely the new contribution we describe is important in the vicinity of the time-reversal symmetry breaking phase transition, that can occur at temperatures much lower than $T_{c}$, where the usual contribution due to thermal quasiparticles is typically extinct. To illustrate this new effect we consider time-reversal symmetry breaking states which are widely expected to exist in iron pnictide compounds [8-11].

A fundamentally interesting state that can appear in multicomponent superconducting systems is the so called $s+i s$ state. In addition to the usual gauge symmetry $U(1)$, it is characterized by a broken time-reversal symmetry (BTRS). Not only is it the simplest BTRS extension of the most abundant $s$-wave state, but also it is expected to arise from various microscopic physics $[8,9,12-15]$. The $s+i s$ state can as well be fabricated on demand on the interfaces of superconducting bilayers [16]. Recently it was demonstrated that such physics very likely occurs in strongly hole doped $\mathrm{Ba}_{1-x} \mathrm{~K}_{x} \mathrm{Fe}_{2} \mathrm{As}_{2}$ $[8,15]$ as well as in stoichiometric compound LiFeAs [9].

In iron pnictides, the $s+i s$ BTRS state originates from the multiband character of superconductivity and several competing pairing channels, as shown schematically on the inset in Fig. 1(a). A typical band structure of $\mathrm{Ba}_{1-x} \mathrm{~K}_{x} \mathrm{Fe}_{2} \mathrm{As}_{2}$ consists of two hole pockets at the $\Gamma$ point and two electron pockets at $(0, \pi)$ and $(\pi, 0)$. The superconducting coupling here is dominated by the interband repulsion between electron and hole Fermi surfaces, as well as between the two hole pockets at $\Gamma$. Such a system can be described by a minimal three-band model, assuming that the order parameter components are $\Delta_{1}$ for electron pockets and $\Delta_{2,3}$ for the hole bands at $\Gamma$ [8]. The competition of repulsion forces results in an intrinsically complex order parameter whose components in each band $\Delta_{k}=\left|\Delta_{k}\right| e^{i \theta_{k}}$ (where $k=1,2,3$ is the band index) possess non-trivial (frustrated) ground-state phase differences $\theta_{k j}=\theta_{k}-\theta_{j}$ which are neither 0 nor $\pi$ $[8,12-15,17]$. The ground-state order parameter of the $s+i s$ phase is not invariant under the discrete $\left(\mathbb{Z}_{2}\right)$ timereversal transformation associated with complex conjugation $\mathcal{T}\left(\Delta_{k}\right)=\Delta_{k}^{*}$. Thus it has a broken $U(1) \times \mathbb{Z}_{2}$ symmetry [17] and below, $s \pm i s$ will stand for states that are related by time-reversal transformation $\mathcal{T}$. One of the interband phase differences, e.g. $\theta_{12}$ can be used as the order parameter that characterizes the $\mathbb{Z}_{2}$ phase transition.

The BTRS states with phase frustration $\theta_{k j} \neq 0, \pi$ imply the existence of persistent "intrinsic Josephson-like currents" between the three bands, as shown schematically in Fig. 1(b,c). These currents have opposite "directions" for the two ground states with opposite values of interband phase differences. Although the underlying microscopic physics is not exotic, nor is any fine tuning is required to form such a state, no experimental observations of such BTRS states have been reported. The reason being the major challenges to distinguish the $s+i s$ state from its time-reversal invariant $s_{ \pm}$and $s_{++}$cousins by conventional methods. Indeed, the probe of relative phases between components of the order parameter in different bands is a non-trivial task. Few proposals have recently emerged for the indirect observation of BTRS 
(a)

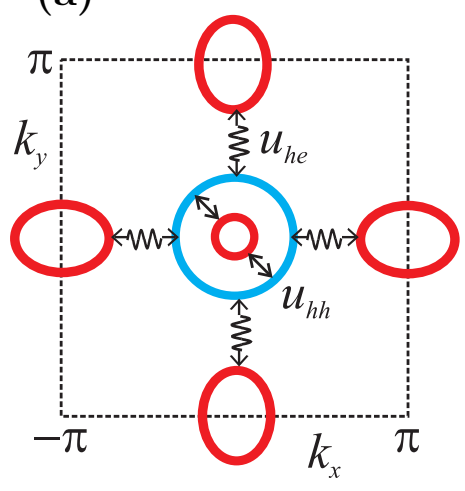

(b)
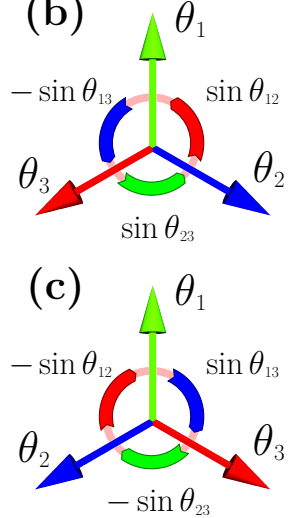

(d)

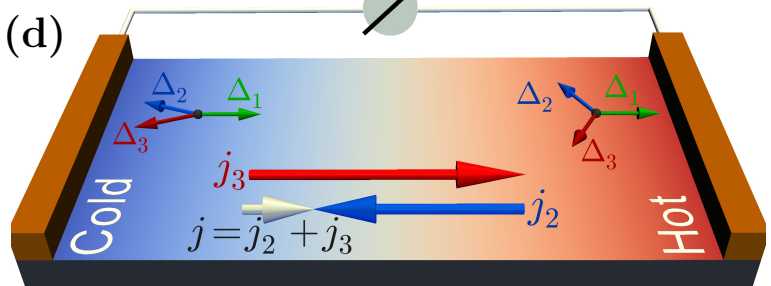

(e)

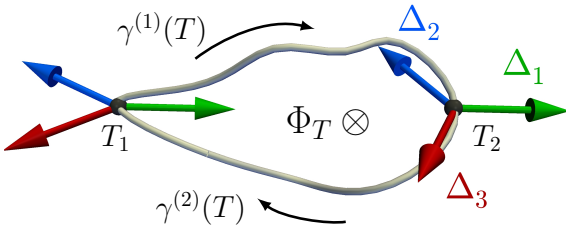

Figure 1. (Color online) - (a) Schematic view of the band structure in hole-doped iron pnictide compound $\mathrm{Ba}_{1-x} \mathrm{~K}_{x} \mathrm{Fe}_{2} \mathrm{As}_{2}$. It consists of two hole pockets at $\Gamma$ shown by open circles and two electron pockets at $(0, \pi)$ and $(\pi, 0)$ shown by ellipses. The s+is state is favored by superconducting coupling dominated by the interband repulsion between electron and hole Fermi surfaces, as well as between the two hole pockets. Panels $(\mathrm{b}, \mathrm{c})$ schematically show the two degenerate ground-states $s \pm i s$. The phases of the order parameter components $\Delta_{k}=\left|\Delta_{k}\right| e^{i \theta_{k}}$ are represented by vector diagrams. Circular arrows show the directions of interband Josephson current supported by non-trivial relative phases $\theta_{k j} \neq 0, \pi$. (d) Sample whose ends are maintained at different temperatures $T_{1,2}$. The thermophase effect appears due to the temperature-dependent interband phase difference $\theta_{k j}=\theta_{k j}(T)$. The total thermally induced current $\boldsymbol{j}$ will have opposite directions in $s \pm i s$ states. Panel (e) sketches a closed circuit in spatially inhomogeneous bulk superconductor with two branches characterized by different values of the thermophase coefficients $\gamma^{(1,2)}(T)$. The junctions between branches have different temperatures $T_{1,2}$ and the temperature bias generates magnetic flux through the circuit (7).

signatures in collective mode spectrum [15, 17-22], unusual topological defects $[23,24]$ or spontaneous currents near impurities in samples subjected to strain [25].

The challenges associated with the observation of the $s+i s$ state and its relevance to pnictides motivate our choice of this kind of material for demonstration of the unusual thermoelectric properties of BTRS superconductors. Below we show that in such systems, there is a new mechanism for thermal generation of supercurrent. It is not related to normal thermal currents and leads to thermoelectric properties which strongly differ from that suggested by Ginzburg, in usual superconductors. Although the suggested mechanism does not eliminate the usual thermoelectric effect, it will be shown to dominate near the BTRS phase transition thus providing an experimental test for this unconventional state.

The key idea behind the proposed thermoelectric effect is that, due to the generically temperature-dependent interband phase differences, i.e., $\theta_{k j}=\theta_{k j}(T)$, a temperature bias generates phase gradients of condensate components. Assuming that temperature gradients are small, so that the order parameter is determined by the local temperature, yields the relation

$$
\nabla \theta_{k}=\nabla \varphi_{0}+\gamma_{k}(T) \nabla T,
$$

where $\varphi_{0}=\sum_{k=1}^{3} \theta_{k} / 3$, and $\gamma_{k}(T)=\frac{1}{3} \sum_{j \neq k} d \theta_{k j} / d T$ are the thermophase coefficients. Superconducting currents can be generated, as a results of this thermophase effect. In multiband superconductors, the total current has contributions from each band $\boldsymbol{j}=\sum_{k} \boldsymbol{j}_{k}$. In units where $\hbar=c=1$, the partial currents read as $\boldsymbol{j}_{k}=\left(\boldsymbol{\nabla} \theta_{k}-2 \pi \boldsymbol{A} / \Phi_{0}\right) / 8 e \pi \lambda_{k}^{2}$, where $\lambda_{k}$ are characteristic constants associated with contributions of a given band to the London penetration depth. $\boldsymbol{A}$ is the vector potential of the magnetic field $\boldsymbol{B}=\boldsymbol{\nabla} \times \boldsymbol{A}$ and $\Phi_{0}$ the flux quantum. Introducing the notation $\boldsymbol{Q}_{0}=\boldsymbol{\nabla} \varphi_{0}-2 \pi \boldsymbol{A} / \Phi_{0}$ and the London penetration length $\lambda_{L}^{2}=1 / \sum_{k} \lambda_{k}^{-2}$, allows to write the total current as

$$
\boldsymbol{j}=\frac{\boldsymbol{Q}_{0}}{8 e \pi \lambda_{L}^{2}}+\sum_{k>i} \frac{\lambda_{i}^{2}-\lambda_{k}^{2}}{24 e \pi \lambda_{k}^{2} \lambda_{i}^{2}} \nabla \theta_{k i} .
$$

The first term here is a usual Meissner current while the second part is determined by the gradients of interband phase differences. It describes the charge transfer by counter-currents of several superconducting components. Observe that according to Eq. (1), the counterflow is generated by the temperature gradient and contributes to the current according to the following generic expression:

$$
\begin{aligned}
\boldsymbol{j} & =\frac{\boldsymbol{Q}_{0}+\gamma(T) \boldsymbol{\nabla} T}{8 e \pi \lambda_{L}^{2}(T)} \\
\gamma(T) & =\lambda_{L}^{2}(T) \sum_{k} \gamma_{k}(T) \lambda_{k}^{-2}(T) .
\end{aligned}
$$

The essence of such a thermophase effect is schematically shown in Fig. 1(d), for the case of $\boldsymbol{Q}_{0}=0$ so that, according to Eq. (1), the partial currents in each band are given 
by $\boldsymbol{j}_{k}=\gamma_{k}(T) \nabla T / 8 e \pi \lambda_{k}^{2}$. Since the thermophase coefficients are opposite for the $s \pm i s$ states: $\mathcal{T} \gamma_{k}=-\gamma_{k}$, these thermally induced superconducting currents are sensitive to time-reversal symmetry transformation. That is, for a given temperature bias, the current directions are opposite in $s+i s$ and in $s-i s$ states.

Deep in the bulk of a superconducting sample the total current (3) should vanish: $\boldsymbol{j}=0$, which yields the condition for current compensation

$$
\boldsymbol{Q}_{0}=-\gamma \nabla T
$$

This compensation can be achieved locally even for inhomogeneous superconductor if the thermophase coefficient has slow spatial dependence $\gamma=\gamma(T, \boldsymbol{r})$. Integrating Eq. (5) along a closed path and assuming for simplicity that no vortices are trapped in the circuit $\left(\oint \boldsymbol{d} \boldsymbol{\ell} \cdot \boldsymbol{\nabla} \varphi_{0}=0\right)$, we get the thermally induced magnetic flux

$$
\Phi_{T}=\frac{\Phi_{0}}{2 \pi} \oint \boldsymbol{d} \ell \cdot \nabla T \gamma(T, \boldsymbol{r})
$$

Since the coefficient $\gamma(T, \boldsymbol{r})$ is in general spatially inhomogeneous along the integration path, the expression (6) yields a finite value of $\Phi_{T}$.

Now consider a closed circuit with two branches that are made up of different superconductors, for example the $\mathrm{Ba}_{1-x} \mathrm{~K}_{x} \mathrm{Fe}_{2} \mathrm{As}_{2}$ compound with different doping levels. The thermophase coefficient $\gamma(T)$ in this case has a step-wise discontinuity along the circuit, determined by the values $\gamma^{(1,2)}(T)$ at the different branches. This is shown schematically in Fig. 1(e). Assuming that the junctions between branches have different temperatures $T_{1,2}$, Eq. (6) yields an induced magnetic flux through the circuit given by

$$
\Phi_{T}=\frac{\Phi_{0}}{2 \pi} \int_{T_{1}}^{T_{2}} d T\left[\gamma^{(1)}(T)-\gamma^{(2)}(T)\right]
$$

The maximal possible magnitude of $\Phi_{T}$ can be estimated by considering one of the branches to be in the timereversal invariant state, so that e.g. $\gamma^{(2)}(T)=0$ and $\lambda_{1} \ll \lambda_{2}$. Then, from Eq. (7), we obtain $\Phi_{T} / \Phi_{0} \approx$ $\delta \theta_{12} / 4 \pi$ where $\delta \theta_{12}=\theta_{12}\left(T_{2}\right)-\theta_{12}\left(T_{1}\right)$.

The resulting thermally induced flux can have a giant magnitude compared to that produced by a usual thermoelectric effect. Below, we will introduce a realistic microscopic model for the $s+i s$ state, to demonstrate that the interband phase difference can have a significant variation as a function of temperature $\max \left(\delta \theta_{12}\right) \sim \pi$. Therefore the resulting flux $\Phi_{T} \sim \Phi_{0}$ is much larger than the typical value of $\Phi_{T} \sim 10^{-2} \Phi_{0}$ expected in usual superconducting thermoelectric circuits $[1,2,26-28]^{1}$.

1 The magnitude of quasiparticle thermoelectric effects in su-
To provide a microscopic basis for this physics, we now proceed to calculate thermophase coefficients for the $s+i s$ superconducting state in $\mathrm{Ba}_{1-x} \mathrm{~K}_{x} \mathrm{Fe}_{2} \mathrm{As}_{2}$. Within the minimal three-band model, it is parametrized by two pairing constants characterizing the strength of interband hole-hole $u_{h h}$ and electron-hole $u_{h e}$ repulsions (as shown schematically in Fig. 1). The self-consistency equation for the order parameter components $\boldsymbol{\Delta}=\left(\Delta_{1}, \Delta_{2}, \Delta_{3}\right)$ $[8,15,21]$

$$
\boldsymbol{\Delta}=2 \pi T \hat{\Lambda} \sum_{n=0}^{N_{d}} \boldsymbol{F}\left(\omega_{n}\right)
$$

where $\boldsymbol{F}=\left(F_{1}, F_{2}, F_{3}\right)$ and $F_{k}\left(\omega_{n}\right)=\Delta_{k} / \sqrt{\omega_{n}^{2}+\left|\Delta_{k}\right|^{2}}$, $\omega_{n}=(2 n+1) \pi T$ are fermionic Matsubara frequencies, $T$ is the temperature. Here $N_{d}=\Omega_{d} /(2 \pi T)$ is a cut-off at Debye frequency, and the coupling parameter matrix is chosen in the form

$$
\hat{\Lambda}=-\left(\begin{array}{ccc}
0 & u_{h h} & u_{h e} \\
u_{h h} & 0 & u_{h e} \\
u_{h e} & u_{h e} & 0
\end{array}\right),
$$

which yields the phase frustration for certain parameters $u_{h h}, u_{h e}$. In this case $\Delta_{1,2}$ and $\Delta_{3}$ describe the order parameter in hole bands and electronic pockets correspondingly. The order parameter has symmetric form $\boldsymbol{\Delta}=e^{i \varphi_{0}}\left(\left|\Delta_{1}\right| e^{i \theta_{12}},\left|\Delta_{2}\right| e^{-i \theta_{12}},\left|\Delta_{3}\right|\right)$, where $\varphi_{0}$ is the common phase and the non-trivial interband phase difference $\theta_{12} \neq 0, \pi$ appears in the TRSB $s+i s$ state. The phase diagram, shown in Fig. 2, is obtained by solving a nonlinear self-consistency problem $(8,9)$. It demonstrates the line of $\mathbb{Z}_{2}$ BTRS phase transition that occurs at the temperature $T_{\mathbb{Z}_{2}} \leq T_{c}$. Within this model the thermophase coefficients in Eq. (3) are given by $\gamma_{1}(T)=-\gamma_{2}(T)=-\frac{1}{2} d \theta_{12} / d T$ and $\gamma_{3}=0$. Despite the symmetry between bands the net thermophase coefficient (4) is in general non-zero since the coefficients $\lambda_{k}$ depend not only on the gap amplitudes but also on the kinetic coefficients. For example in the diffusive limit they are given by a standard text-book expression for the London length $\lambda_{k}^{-2}=4 \pi^{2} \sigma_{k}\left|\Delta_{k}\right| \tanh \left(\left|\Delta_{k}\right| / 2 T\right)$ where $\sigma_{k}$ are the normal state conductivities in each band which are different even if the superconducting pairing is symmetric between hole bands. In the vicinity of $T_{\mathbb{Z}_{2}}$ the thermophase coefficients diverge as $\gamma(T) \sim\left(T_{\mathbb{Z}_{2}}-T\right)^{-1 / 2}$. In Fig. 2(b) the overall profile of the function $\left(T_{\mathbb{Z}_{2}}-T\right) \gamma(T)$ is shown, where the prefactor $\left(T_{\mathbb{Z}_{2}}-T\right)$ is added to remove the divergence. The typical temperature dependencies of $\gamma(T)$ are shown in Fig. 2(c) for particular coupling parameters.

perconductors is still a challenging problem, see discussion in Ref. [29]. It is beyond the scope of the present paper since we deal with unconventional thermoelectric effect, which is not related to the flow of quasiparticles. 
The square-root singularity dependence of thermophase coefficient (see Fig. 2c) is consistent with the fact that the effective potential for the phase difference gets soft near the $\mathbb{Z}_{2}$ symmetry-restoring phase transition. However it is in strong contrast with the quasiparticle contribution which has a smooth thermally activated behavior[26, 27].

Hence the thermophase coefficients are large near $T_{\mathbb{Z}_{2}}$ and the discussed thermoelectric effect is generically the dominating one at low temperatures in the fully gapped BTRS $s+i s$ state. The magnitude of the total variation of the relative phase $\theta_{12}$ as a function of temperature can be estimated from the typical dependence in Fig. 2(c). There $\delta \theta_{12} \approx 0.4 \pi$, so that the thermally generated flux is $\Phi_{T} \sim 0.2 \Phi_{0}$ which is in general much larger than the theoretically predicted value for quasiparticle contribution.

To draw a more convincing comparison let us calculate the thermoelectric fluxes in the BTRS state $\Phi_{T}^{B T R S}$ and in the usual time-reversal symmetric superconductor $\Phi_{T}^{T R S}$ generated in the thermoelectric circuit according to Eq.(7). For simplicity we assume that one of the branches is passive $\gamma_{2}=0$ and considerer the tempera-
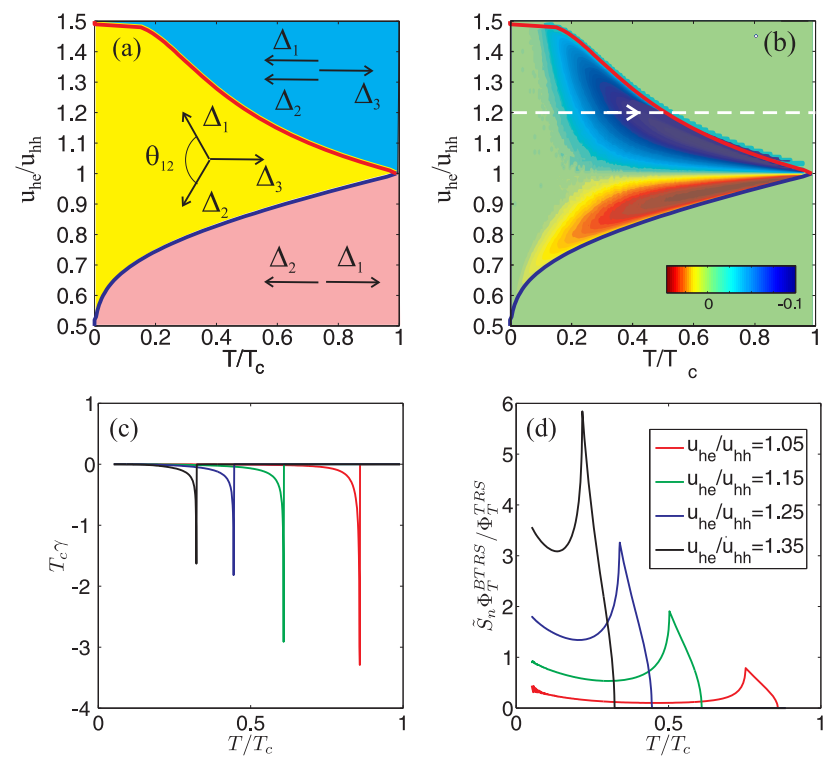

Figure 2. (Color online) - (a) Phase diagram of the threeband superconductor described by the microscopic model (see text). Solid curves show the critical temperature $T_{\mathbb{Z}_{2}}$ of the BTRS phase transition. (b) Color scale plot of the thermophase coefficient $\left(T_{\mathbb{Z}_{2}}-T\right) \gamma(T)$. The prefactor $\left(T_{\mathbb{Z}_{2}}-T\right)$ is added to regularize the square-root singularity of $\gamma(T)$ at the BTRS phase transition. (c) The temperature dependencies of $\gamma(T)$ along the lines such as shown by arrow in the panel (b). From right to left $u_{h e} / u_{h h}=1.05 ; 1.15 ; 1.25 ; 1.35$. (d) The ratio of magnetic fluxes generated due to the unconventional and conventional thermoelectric effects, $\Phi_{T}^{B T R S}$ and $\Phi_{T}^{T R S}$ correspondingly. $\tilde{S}_{n}$ is a dimensionless normal state Seebeck coefficient which can be of the order 1 but typically is much smaller. The other system parameters are explained in text. ture bias $T_{2}-T_{1}=0.1 T_{c}$ which is of the order of $1-5 \mathrm{~K}$ in iron pnictides. To calculate a usual quasiparticle contribution we use the result of Refs.[26, 27]: $\gamma=4 \pi \lambda_{L}^{2} \eta$ where $\eta=0.6 \sigma_{n} \tilde{S}_{n}\left(T / T_{c}\right) \int_{\Delta_{0} / T}^{\infty} d y y^{2} \cosh ^{-2}(y / 2)$. Here $\Delta_{0}$ is the smallest gap in the system, $\sigma_{n}$ is the normal state conductivity and $\tilde{S}_{n}=\left(e / k_{B}\right) S_{n}$ where $S_{n}$ is a Seebeck coefficient just above $T_{c}, k_{B}$ is Boltzmann constant and $e$ is the electron charge. We use a diffusive model for $\lambda_{k}$ parametrized by kinetic coefficients $\sigma_{3}=\sigma_{2}=\alpha \sigma_{1}$ where the coefficient $\alpha \sim 1$ measures an asymmetry between hole pockets. Note that within our simplified model of equal coupling constants for the hole bands the overall magnitude of the unconventional thermoelectric effect is proportional to the asymmetry of kinetic coefficients $\sigma_{1}-\sigma_{2}=(\alpha-1) \sigma_{1}$, which we assume below to be rather small $\alpha=0.9$. For such parameters the typical temperature dependencies of the ratio $\tilde{S}_{n} \Phi_{B T R S} / \Phi_{T R S}$ are shown in Fig. 2(d). From this plot one can see that even for quite a large values of Seebeck coefficient $S_{n} \sim 100 \mu V / K$ and hence $\tilde{S}_{n} \sim 1$ observed in some iron-oxipnictide compounds [30] we obtain that the unconventional thermoelectric signal is in fact much larger than the quasiparticle contribution especially if $T_{Z 2} \ll T_{c}$, see black curve in Fig. 2(d).

The usual thermoelectric effect can be significant at low temperatures if quasiparticle density remains finite, e.g., in gapless superconductors. However, we would like to emphasize that even in this case the suggested effect can be separated from quasiparticle contribution since it breaks the time-reversal symmetry. That is, the direction of thermally induced magnetic field is different in $s+i s$ and $s-i s$ states while the usual thermoelectric effect is time-reversal invariant.

Let us now consider several characteristic examples which should arise in concrete experimental set-ups based on circuits containing BTRS $s+i s$ superconductors. To that end, we use the three-component Ginzburg-Landau (GL) theory (10), although the effects will be similar in the microscopic formalism. The GL expansion, derived from the microscopic three-band model (see Appendix for details), reads as

$$
\begin{aligned}
\mathcal{F} & =\sum_{k=1}^{3} \frac{D_{k}}{2}\left|\left(\boldsymbol{\nabla}+i \frac{2 \pi}{\Phi_{0}} \boldsymbol{A}\right) \Delta_{k}\right|^{2}+\alpha_{k}\left|\Delta_{k}\right|^{2}+\frac{\beta_{k}}{2}\left|\Delta_{k}\right|^{4} \\
& +\sum_{k=1}^{3} \sum_{j>k}^{3} \eta_{k j}\left|\Delta_{k}\right|\left|\Delta_{j}\right| \cos \theta_{k j}+\frac{\boldsymbol{B}^{2}}{2}
\end{aligned}
$$

where $j, k$ are band indices, and $D_{k}$ are diffusion constants. We model temperature dependence of the coefficients as $\alpha_{k} \simeq \alpha_{k}^{0}\left(T / T_{k}-1\right)\left(\alpha_{k}^{0}\right.$ and $T_{k}$ being characteristic constants, further details on the derivation and choice of parameters are given in Appendix ). The numerical studies of the GL equations are performed within a finite-element formulation [31] and using a non-linear conjugate gradient algorithm, with the standard condi- 


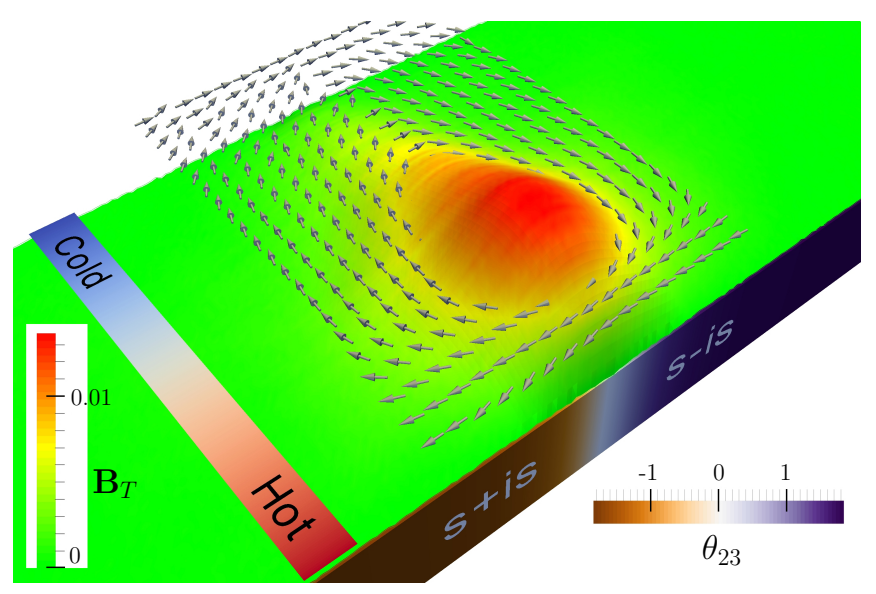

Figure 3. (Color online) - Thermally induced magnetic field on a domain-wall between $s+i s$ and $s-i s$ phases. Two faces of the stripe are maintained at different temperatures, thus resulting in a thermal gradient, here $\delta T=0.2 T_{c}$, along the domain-wall. The $s+i s / s-i s$ domain-wall generates the magnetic field $\mathbf{B}_{T}$. In the absence of the temperature gradient a domain-wall in $s+i s$ superconductor does not have any magnetic signature.

tion that no current flows through the sample's boundaries.

First, we consider thermoelectric response of a sample with a domain wall that separate $s \pm i s$ phases, which are natural inhomogeneities expected to exist in realistic systems with spontaneously broken discrete symmetries. It is known that such domain walls do not carry magnetic field at constant temperature [24]. We find that, in the presence of a temperature gradient along the domain wall, it generates a thermally induced magnetic field, as shown in Fig. 3. There, the thermophase coefficients $\gamma(T)$ have opposite signs in $s+i s / s-i s$ domains. Therefore, in the vicinity of the interface between these, there should be net superconducting current and a thermally induced magnetic field $\boldsymbol{B}_{T}$. Note that there is no phase winding in this flux-carrying configuration and the thermally induced flux $\boldsymbol{B}_{T}$ is not quantized ${ }^{2}$. Since the condensate density near domain wall is non-homogeneous one cannot avoid additional signal produced by usual the thermoelectric effect according to Ginzburg's mechanism. However, it generates a dipole-like magnetic field distribution

2 Magnetic flux penetration into a superconductor without phase winding/vortices is not restricted to three-band superconductors. This can be seen from the general relation for magnetic flux obtained from the expression supercurrent $\Phi=\oint \boldsymbol{A} d \ell=$ $\oint\left[\boldsymbol{J} / \rho(\mathbf{r})+\sum_{i=1 . . N}\left|\Delta_{i}(\mathbf{r})\right|^{2} \nabla \theta_{i}(\mathbf{r}) / \rho(\mathbf{r})\right](\rho(\mathbf{r})$ being the total density). Even if there is no phase winding and even if we select the integration contour where $\boldsymbol{J} \rightarrow 0$, the integral will in general be nonzero, in the presence of gradients of relative phases $\theta_{i}-\theta_{j}$ and densities. which does not affect the total thermally induced magnetic flux. For a homogeneous BTRS state in the absence of domain walls both normal and unconventional thermoelectric signals are absent.

The non-trivial thermoelectric effect in $s+i s$ phases can be employed to obtain an advanced functionality in the practical applications of certain iron-based superconductors. For this purpose one can manipulate the symmetry of superconducting states by simultaneously applying temperature bias and an external magnetic field $\boldsymbol{H}_{\text {ext }}$ which in general removes the degeneracy of $s+i s / s-i s$ states. As a result it is possible to prepare a particular state by cooling the system through a BTRS transition. As an example we suggest a scheme of a memory cell based on the non-trivial thermoelectric properties of bimetallic rings shown in Fig. 4 with both branches made of $s+i s$ superconductor, but having different doping. The junctions between branches are maintained at different temperatures $T_{1,2}$ as sketched in Fig. 4(a). There, the $s \pm i s$ states have the opposite val-
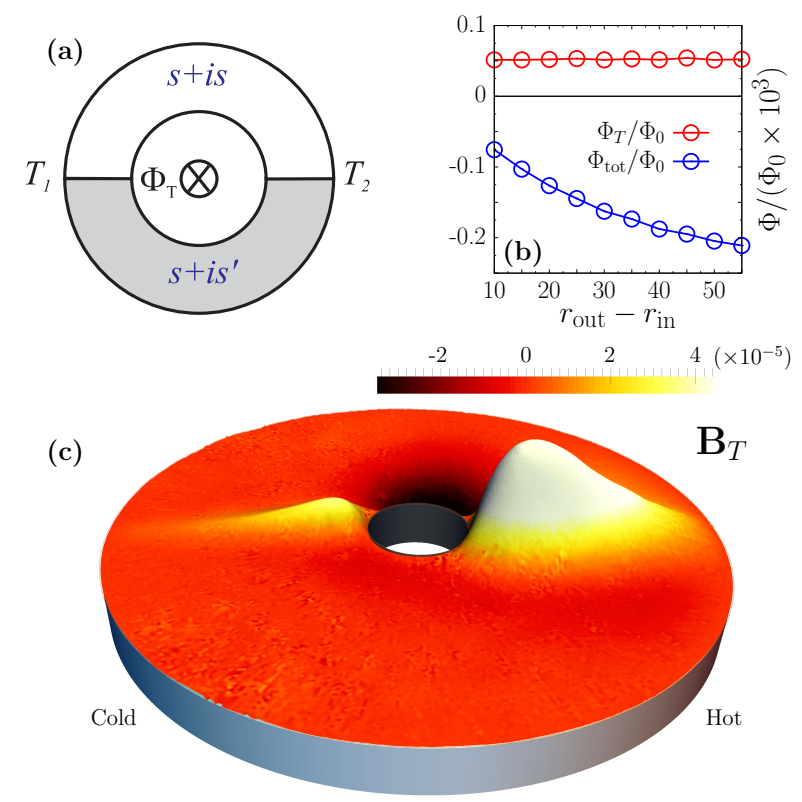

Figure 4. (Color online) - A bimetallic ring consisting of two branches in the $s \pm i s$ state, but having different chemical composition (see details in Appendix ). Both junctions are maintained at different temperatures $T_{1,2}$, so that there is a thermal gradient that varies linearly with the polar angle. This is sketched in panel (a). The resulting thermally induced magnetic field is shown on panel (c). In contrast to the usual thermoelectric effect its overall sign depends not only on the temperature bias but also on which of $\mathbb{Z}_{2}$ BTRS ground states (i.e. $s+i s$ or $s-i s$ ) is realized. This configuration supports nonzero flux $\Phi_{T}$ in the inner hole, which is more or less independent of the diameter of the inner hole. The total flux, on the other hand depends on the diameter of the sample (b). 
ues of $\boldsymbol{B}_{T}{ }^{3}$. In such a system the value of the $\mathbb{Z}_{2}$ index, which distinguishes $s \pm i s$ states, can be considered as a bit of information. Let us now describe the writing/reading protocols. First the external field $\boldsymbol{H}_{\text {ext }}$ interacts with thermally induced currents and removes the $s \pm i s$ degeneracy. Hence the particular $s+i s$ or $s-i s$ state can be selected when such a system is cooled through the BTRS phase transition, in the presence of both external field and temperature bias $\delta T=T_{2}-T_{1}$. After cooling, both the external field and temperature bias can be removed, leaving the system in the ground state characterized by the $\mathbb{Z}_{2}$ index $\operatorname{sgn}\left(\delta T H_{\text {ext }}\right)$. The read-out protocol, i.e. the measurement of $\mathbb{Z}_{2}$ index, can be implemented by applying the temperature bias (in the absence of external field) and measuring the direction of induced magnetic flux.

To conclude, we demonstrated that multicomponent superconductors with broken time-reversal symmetry, and in particular the $s+i s$ state, feature in the vicinity of BTRS transition, a giant thermoelectric effect of principally different nature than that in single-component superconductors. It originates in thermally induced intercomponent counter-flows, in contrast to the counter-flows of normal and superconducting currents in the mechanism originally discussed by Ginzburg. Although related effects should be present in various multicomponent superconductors, along with multicomponent superfluids, we focused on the $s+i s$ states where the effect is generic (irrespective of the model).

The work was supported by the Swedish Research Council grants 642-2013-7837, 325-2009-7664. The computations were performed on resources provided by the Swedish National Infrastructure for Computing (SNIC) at National Supercomputer Center at Linköping, Sweden.

3 The unconventional pattern of magnetic field induced by counter-flows is related to the general property of non-Meissner contribution to electrodynamics, in multicomponent superconductors. The essence of the physics leading to the Meissner and, in a broader sense, to the Anderson-Higgs effect is that the magnetic field is described by a massive vector field theory. As shown in Refs. [32, 33], multicomponent systems cannot generically be described by a massive vector field theory. Rather, it can be mapped onto a massive vector field theory coupled to another field associated with the charge-carrying counter-flows. The later terms lead to contributions which are typically negligible or unobservable in ordinary multiband superconductors due for example to interband couplings. By contrast as discussed in this paper, the thermoelectric effect in the $s+i s$ state, makes the interband phase fluctuations propagate into the bulk. This produces the unconventional magnetic signatures.

\section{Ginzburg-Landau expansion for multiband superconductors}

To derive the Ginzburg-Landau expansion (10), we use the microscopic self-consistency equation

$$
\boldsymbol{\Delta}=2 \pi T \hat{\Lambda} \sum_{n=0}^{N_{d}} \boldsymbol{F}\left(\omega_{n}\right),
$$

where $\boldsymbol{F}=\left(F_{1}, F_{2}, F_{3}\right)$ and $F_{k}\left(\omega_{n}\right)=\Delta_{k} / \sqrt{\omega_{n}^{2}+\left|\Delta_{k}\right|^{2}}$. $\omega_{n}=(2 n+1) \pi T$, where $n \in \mathbb{Z}$ is the fermionic Matsubara frequency, $T$ the temperature, and $N_{d}=\Omega_{d} /(2 \pi T)$ is a cut-off at Debye frequency. In the diffusive case the anomalous functions in each band can be expanded as follows:

$$
F_{j}=\frac{\Delta_{j}}{\omega_{n}}-\frac{\Delta_{j}\left|\Delta_{j}\right|^{2}}{2 \omega_{n}^{3}}+\frac{D_{j}(\boldsymbol{\nabla}-i e \boldsymbol{A})^{2} \Delta_{j}}{2 \omega_{n}^{2}}
$$

where $D_{j}$ is the diffusion coefficient. Then we get for the summation over Matsubara frequencies

$$
\begin{aligned}
2 \pi T \sum_{n=0}^{\infty} 1 / \omega_{n} & =G_{0}+\tau, \\
2 \pi T \sum_{n=0}^{\infty} 1 /\left(2 \omega_{n}^{3}\right) & =0.1 / T^{2}, \\
2 \pi T \sum_{n=0}^{\infty} 1 /\left(2 \omega_{n}^{2}\right) & =0.4 / T
\end{aligned}
$$

where $\tau=\left(1-T / T_{c}\right)$. We normalize $\Delta$ by $T_{c}$ and $\xi_{0}=\sqrt{\pi D_{0} / 8 T_{c}}$, where $D_{0}$ is some arbitrary diffusion constant.

$$
\left(G_{0}+\tau-\hat{\Lambda}^{-1}\right) \boldsymbol{\Delta}-b|\boldsymbol{\Delta}|^{2} \cdot \boldsymbol{\Delta}=\tilde{\boldsymbol{D}} \cdot\left(\boldsymbol{\Pi}^{2} \boldsymbol{\Delta}\right)
$$

where $b=0.1, \boldsymbol{\Pi}=\boldsymbol{\nabla}-i e \boldsymbol{A}, \boldsymbol{\Delta}=\left(\Delta_{1}, \Delta_{2}, \Delta_{3}\right),|\boldsymbol{\Delta}|^{2}=$ $\left(\left|\Delta_{1}\right|^{2},\left|\Delta_{2}\right|^{2},\left|\Delta_{3}\right|^{2}\right)$, and $\tilde{\boldsymbol{D}}=\left(\tilde{D}_{1}, \tilde{D}_{2}, \tilde{D}_{3}\right)$ where $\tilde{D}_{j}=$ $D_{j} / D_{0}$. The diffusion coefficients can be different. In the diffusive case the mixed gradient terms are absent.

Let us consider the case of the intraband dominated pairing which can be described by a three-component GL theory in the vicinity of $T_{c}$. This regime is described by the following coupling matrix:

$$
\hat{\Lambda}=\left(\begin{array}{ccc}
\lambda & -\eta_{h} & -\eta_{e} \\
-\eta_{h} & \lambda & -\eta_{e} \\
-\eta_{e} & -\eta_{e} & \lambda
\end{array}\right)
$$

where $\eta_{e}, \eta_{h} \ll \lambda$. The critical temperature is determined by the equation $G_{0}=\min \left(\lambda_{1}^{-1}, \lambda_{2}^{-1}, \lambda_{3}^{-1}\right)$, where $\lambda_{1,2}^{-1}=$ $\left(2 \lambda-\eta_{h} \pm \sqrt{8 \eta_{e}^{2}+\eta_{h}^{2}}\right) /\left[2\left(\lambda^{2}-\lambda \eta_{h}-2 \eta_{e}^{2}\right)\right]$ and $\lambda_{3}^{-1}=$ $1 /\left(\lambda+\eta_{h}\right)$ are the positive eigenvalues of the matrix

$$
\hat{\Lambda}^{-1}=X\left(\begin{array}{ccc}
\lambda^{2}-\eta_{e}^{2} & \eta_{e}^{2}+\lambda \eta_{h} & \eta_{e}\left(\lambda+\eta_{h}\right) \\
\eta_{e}^{2}+\lambda \eta_{h} & \lambda^{2}-\eta_{e}^{2} & \eta_{e}\left(\lambda+\eta_{h}\right) \\
\eta_{e}\left(\lambda+\eta_{h}\right) & \eta_{e}\left(\lambda+\eta_{h}\right) & \lambda^{2}-\eta_{h}^{2}
\end{array}\right)
$$


where $X=1 /\left[\left(\lambda^{2}-\lambda \eta_{h}-2 \eta_{e}^{2}\right)\left(\lambda+\eta_{h}\right)\right]$. Since we assume that $\eta_{e, h}>0$ and $\eta_{e, h} \ll \lambda$ the critical temperature is given by the smallest eigenvalue $G_{0}=1 /\left(\lambda+\eta_{h}\right)$ so that

$$
G_{0} \hat{I}-\hat{\Lambda}^{-1}=-\left(\begin{array}{ccc}
a_{1} & a_{1} & a_{2} \\
a_{1} & a_{1} & a_{2} \\
a_{2} & a_{2} & a_{3}
\end{array}\right)
$$

where

$$
\begin{aligned}
& a_{1}=\left(\eta_{e}^{2}+\lambda \eta_{h}\right) / X \\
& a_{2}=\eta_{e}\left(\lambda+\eta_{h}\right) / X \\
& a_{3}=\left(2 \eta_{e}^{2}-\eta_{h}^{2}+\lambda \eta_{h}\right) / X .
\end{aligned}
$$

For example, for $\lambda=1$ and $\eta_{e}=0.1, \eta_{h}=0.2$ we get $a_{1}=0.2244, a_{2}=0.1282$ and $a_{3}=0.1923$. For $\lambda=1$ and $\eta_{e}=\eta_{h}=0.1$ we get $a_{1}=a_{2}=a_{3}=0.1136$.

Then we get the system of GL equations

$$
\begin{aligned}
& \left(\tau-a_{1}\right) \Delta_{1}-a_{1} \Delta_{2}-a_{2} \Delta_{3}-b \Delta_{1}\left|\Delta_{1}\right|^{2}+\tilde{D}_{1} \Pi^{2} \Delta_{1}=0 \\
& \left(\tau-a_{1}\right) \Delta_{2}-a_{1} \Delta_{1}-a_{2} \Delta_{3}-b \Delta_{2}\left|\Delta_{2}\right|^{2}+\tilde{D}_{2} \Pi^{2} \Delta_{2}=0 \\
& \left(\tau-a_{3}\right) \Delta_{3}-a_{2} \Delta_{1}-a_{2} \Delta_{2}-b \Delta_{3}\left|\Delta_{3}\right|^{2}+\tilde{D}_{3} \Pi^{2} \Delta_{3}=0
\end{aligned}
$$

where $b=0.1, \boldsymbol{\Pi}=\boldsymbol{\nabla}-i e \boldsymbol{A}$ and $\tilde{D}_{j}=D_{j} / D_{0}$. The diffusion coefficients can be different. In the diffusive case the mixed gradient terms are absent. The free energy functional whose variations gives the Ginzburg-Landau equations reads as

$$
\begin{aligned}
\mathcal{F}=\frac{\boldsymbol{B}^{2}}{2} & +\sum_{k=1}^{3}\left\{\tilde{D}_{k}\left|\boldsymbol{\Pi} \Delta_{k}\right|^{2}+\alpha_{k}\left|\Delta_{k}\right|^{2}+\frac{\beta_{k}}{2}\left|\Delta_{k}\right|^{4}\right\} \\
& +\sum_{k=1}^{3} \sum_{j=k+1}^{3} \eta_{k j}\left\{\Delta_{k}^{*} \Delta_{j}+\Delta_{j}^{*} \Delta_{k}\right\}
\end{aligned}
$$

where $\beta_{k}=b, \eta_{12}=a_{1}, \eta_{13}=\eta_{23}=a_{2}, \alpha_{k}=\alpha_{k}^{0}\left(T / T_{k}-\right.$ $1)$, and $\alpha_{k}^{0}=1-a_{k}, T_{k}=T_{c}\left(1-a_{k}\right)$.

\section{Details of simulations}

In order to investigate the response to the thermal gradients, we minimize numerically the free energy (19), in zero external field, with the standard condition that no current flows through the sample's boundaries. In simulations we use the microscopically derived coefficients (18). The theory is discretized within a finite element formulation [31] and the minimization is performed using a non-linear conjugate gradient algorithm.

\section{Details for the bimetallic ring}

In Fig. 4 of the main text we consider the case of a bimetallic that consists of two branches in the $s+i s$ state, but with different chemical composition. The difference in the chemical composition is modelled by modulating the Ginzburg-Landau coefficients (18) in the lower branch to be $85 \%$ of their value in the upper branch: $a_{i}^{\text {(lower) }}=0.85 a_{i}^{\text {(upper) }}$. Thus the $a_{i}$ vary stepwise while passing from one branch to the other. The interfaces between both branches are maintained at different temperatures $T_{1}$ and $T_{2}$. Here this is the whole interface that is maintained at a given temperature, and thus the temperature is a linear function of the polar angle. This set-up determines the spatially inhomogeneous coefficients $\alpha_{k}$ and $\eta_{k j}$ used in Fig. 4.

[1] V. L. Ginzburg, "On Thermoelectric Phenomena in Superconductors," J. Phys. USSR 8, 148 (1944), [Zh. Eksp. Teor. Fiz. 14, 177 (1944)].

[2] Vitaly L. Ginzburg, "Nobel Lecture: On superconductivity and superfluidity (what I have and have not managed to do) as well as on the "physical minimum" at the beginning of the XXI century," Rev. Mod. Phys. 76, 981-998 (2004).

[3] F. Giazotto, T. T. Heikkilä, and F. S. Bergeret, "Very Large Thermophase in Ferromagnetic Josephson Junctions," Phys. Rev. Lett. 114, 067001 (2015).

[4] A. Ozaeta, P. Virtanen, F. S. Bergeret, and T. T. Heikkilä, "Predicted Very Large Thermoelectric Effect in Ferromagnet-Superconductor Junctions in the Presence of a Spin-Splitting Magnetic Field," Phys. Rev. Lett. 112, 057001 (2014).

[5] P. Machon, M. Eschrig, and W. Belzig, "Nonlocal Thermoelectric Effects and Nonlocal Onsager relations in a Three-Terminal Proximity-Coupled SuperconductorFerromagnet Device," Phys. Rev. Lett. 110, 047002 (2013).

[6] T. Löfwander and M. Fogelström, "Large thermoelectric effects in unconventional superconductors," Phys. Rev. B 70, 024515 (2004).

[7] D. J. Van Harlingen, D. F. Heidel, and J. C. Garland, "Experimental study of thermoelectricity in superconducting indium," Phys. Rev. B 21, 1842-1857 (1980).

[8] S. Maiti, M. M. Korshunov, and A. V. Chubukov, "Gap symmetry in $\mathrm{KFe}_{2} \mathrm{As}_{2}$ and the $\cos 4 \theta$ gap component in LiFeAs," Phys. Rev. B 85, 014511 (2012).

[9] F. Ahn, I. Eremin, J. Knolle, V. B. Zabolotnyy, S. V. Borisenko, B. Büchner, and A. V. Chubukov, "Superconductivity from repulsion in LiFeAs: Novel $s$-wave symmetry and potential time-reversal symmetry breaking," Phys. Rev. B 89, 144513 (2014).

[10] Wei-Cheng Lee, Shou-Cheng Zhang, and Congjun Wu, "Pairing State with a Time-Reversal Symmetry Breaking in FeAs-Based Superconductors," Phys. Rev. Lett. 102, 217002 (2009).

[11] Christian Platt, Ronny Thomale, Carsten Honerkamp, Shou-Cheng Zhang, and Werner Hanke, "Mechanism for a pairing state with time-reversal symmetry breaking in iron-based superconductors," Phys. Rev. B 85, 180502 (2012).

[12] Valentin Stanev and Zlatko Tešanović, "Three-band superconductivity and the order parameter that breaks time-reversal symmetry," Phys. Rev. B 81, 134522 (2010). 
[13] Ronny Thomale, Christian Platt, Werner Hanke, Jiangping $\mathrm{Hu}$, and B. A. Bernevig, "Exotic $d$-Wave Superconducting State of Strongly Hole-Doped $\mathrm{K}_{x} \mathrm{Ba}_{1-x} \mathrm{Fe}_{2} \mathrm{As}_{2}$," Phys. Rev. Lett. 107, 117001 (2011).

[14] Katsuhiro Suzuki, Hidetomo Usui, and Kazuhiko Kuroki, "Spin fluctuations and unconventional pairing in $\mathrm{KFe}_{2} \mathrm{As}_{2}$," Phys. Rev. B 84, 144514 (2011).

[15] Saurabh Maiti and Andrey V. Chubukov, " $s+i s$ state with broken time-reversal symmetry in Fe-based superconductors," Phys. Rev. B 87, 144511 (2013).

[16] T. K. Ng and N. Nagaosa, "Broken time-reversal symmetry in Josephson junction involving two-band superconductors," Europhysics Letters 87, 17003-+ (2009).

[17] Johan Carlström, Julien Garaud, and Egor Babaev, "Length scales, collective modes, and type- 1.5 regimes in three-band superconductors," Phys. Rev. B 84, 134518 (2011).

[18] G. Blumberg, A. Mialitsin, B. S. Dennis, M. V. Klein, N. D. Zhigadlo, and J. Karpinski, "Observation of Leggett's Collective Mode in a Multiband $\mathrm{MgB}_{2}$ Superconductor," Phys. Rev. Lett. 99, 227002 (2007).

[19] Shi-Zeng Lin and Xiao Hu, "Massless Leggett Mode in Three-Band Superconductors with Time-ReversalSymmetry Breaking," Phys. Rev. Lett. 108, 177005 (2012).

[20] Valentin Stanev, "Model of collective modes in threeband superconductors with repulsive interband interactions," Phys. Rev. B 85, 174520 (2012).

[21] M. Marciani, L. Fanfarillo, C. Castellani, and L. Benfatto, "Leggett modes in iron-based superconductors as a probe of time-reversal symmetry breaking," Phys. Rev. B 88, 214508 (2013).

[22] M. Khodas, A. V. Chubukov, and G. Blumberg, "Collective modes in multiband superconductors: Raman scattering in iron selenides," Phys. Rev. B 89, 245134 (2014).
[23] Julien Garaud, Johan Carlström, and Egor Babaev, "Topological Solitons in Three-Band Superconductors with Broken Time Reversal Symmetry," Phys. Rev. Lett. 107, 197001 (2011).

[24] Julien Garaud and Egor Babaev, "Domain Walls and Their Experimental Signatures in $s+i s$ Superconductors," Phys. Rev. Lett. 112, 017003 (2014).

[25] Saurabh Maiti, Manfred Sigrist, and Andrey Chubukov, "Spontaneous currents in a superconductor with $s+i s$ symmetry," Phys. Rev. B 91, 161102 (2015).

[26] Y. M. Gal'Perin, V. L. Gurevich, and V. I. Kozub, "Acoustoelectric and Thermoelectric Effects in Superconductors," JETP Letters 17, 476 (1973), [Zh. Eksp. Teor. Flz. Pisma Redaktsiiu 17, 687 (1973)].

[27] Y. M. Gal'perin, V. L. Gurevich, and V. I. Kozub, "Thermoelectric effects in superconductors," Sov. Phys. JETP 39, 680 (1974), [Zh. Eksp. Teor. Flz. 66, 1387 (1974)].

[28] N. V. Zavaritskii, "Observation of superconducting current excited in a superconductor by heat flow," JETP Letters 19, 126 (1974), [Zh. Eksp. Teor. Flz. Pisma Redaktsiiu 19, 205 (1974)].

[29] Y. M. Gal'perin, V. L. Gurevich, V. I. Kozub, and A. L. Shelankov, "Theory of thermoelectric phenomena in superconductors," Phys. Rev. B 65, 064531 (2002).

[30] L. Pinsard-Gaudart, D. Bérardan, J. Bobroff, and N. Dragoe, "Large Seebeck coefficients in ironoxypnictides: a new route towards n-type thermoelectric materials," physica status solidi (RRL) - Rapid Research Letters 2, 185-187 (2008).

[31] F. Hecht, "New development in freefem++," J. Numer. Math. 20, 251-265 (2012).

[32] Egor Babaev, L. D. Faddeev, and Antti J. Niemi, "Hidden symmetry and knot solitons in a charged two- condensate Bose system," Phys. Rev. B65, 100512 (2002).

[33] Egor Babaev, "Non-Meissner electrodynamics and knotted solitons in two- component superconductors," Phys. Rev. B 79, 104506 (2009). 